\title{
DIRTMAP: development of a web-based dust archive
}

\author{
Barbara A. Maher and David T. Leedal
}

The influence of airborne dust on climate, through changes in the radiative properties of the atmosphere and through iron fertilization of oceans, remains a poorly quantified element of the Earth's climate system, both in the present and in the past. Key dust properties include fluxes, mineralogy, and particle size and shape. However, dust-cycle models presently employ a relatively simple representation of dust properties (e.g. assuming spherical particles); these simplifications severely limit the realism of simulations of the impact of changes in dust loading on radiative forcing and biogeochemical cycling. One way to overcome this limitation and complement and validate these modeling efforts is to use records of dust deposition to assess what effects dust had in the past under different climatic conditions. This is where DIRTMAP, a dust database tailored for such interdisciplinary mineral dust research, could prove a valuable resource. Recently, the original DIRTMAP database (Kohfeld and Harrison 2001) was re-developed into a readily accessible web-based archive which allows interactive interrogation and visualization of the data.

\section{DIRTMAP's evolution}

The Dust Indicators and Records of Terrestrial and MArine Palaeoenvironments (DIRTMAP1) database was originally developed in Lund, Sweden in 1997, as a product of the Mineral aerosols and glacial-interglacial cycles project, led by Sandy Harrison, Colin Prentice and Henning Rodhe. It was designed to enable mapping of mass accumulation rates (MARs) of dust at the Last Glacial Maximum compared with the late Holocene.

DIRTMAP2, subsequently developed by Karen Kohfeld and colleagues at Jena, Germany, included dust information from more sites, encompassing dust fluxes in ice cores, marine sediments, marine sediment traps, and loess sediments. Archived at the World Data Center for Paleoclimatology (ftp://ftp.ncdc.noaa.gov/pub/data/paleo/ loess/dirtmap/), it also provided information on site age models (e.g. layer counting, radiocarbon, oxygen isotope stratigraphy), methods used for calculation of aeolian accumulation rate (e.g. isolation of terrigenous components, Al concentration measurements, number or mass concentrations), and marine sites with non-aeolian sedimentary contributions (e.g. IRD, sediment redistribution)

Other than the incorporation of some additional loess data, the underlying database for the next version, DIRTMAP3 (Maher et al. 2010), underwent relatively little change, as the multi- and inter-disciplinary dust research communities debated their future aims, methodologies, time-slices, dynamics and environments of enquiry. It became

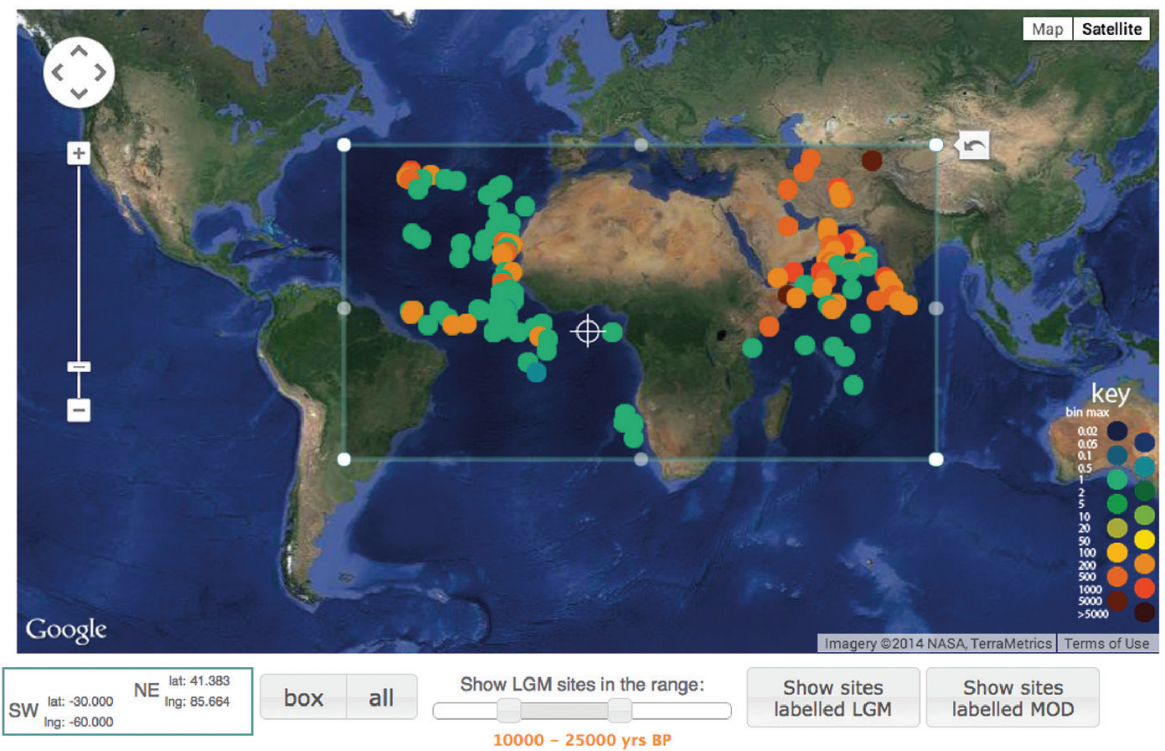

clear that any new version of DIRTMAP had to be not only more easily accessible but also more flexible in order to accommodate more diverse research objectives.

\section{DIRTMAP4}

Funded through the International Union for Quaternary Research (INQUA), the "Dust and Climate Working Group", led by Lancaster University (www.lancaster.ac.uk/lec/sites/ dirtmap/INQU), is currently developing a new website for the development of DIRTMAP4. The aim is to provide the opportunity for rapid updating and quality control of data, and the subsequent development of a new data archiving and access protocol, e.g. enabling archiving of data for additional time slices, and new meta-data fields (e.g. proxy data; particle mineralogy, size distribution, particle shape; bioavailable elements).

As part of this work on DIRTMAP4, we created a web interface to access the database and take advantage of the power of modern browsers and Asynchronous JavaScript and $\mathrm{XML}(\mathrm{AJAX})$ technology, thereby eliminating the need for users to install additional software to access the database. The aim is to provide the dust research community with instant access to the data and allow interactive interrogation and visualization (Fig. 1). A prototype version of the website is presently online at www.lancaster.ac.uk/lec/ sites/dirtmap/hw.html.

The design has intentionally been kept simple to make it easy for other researchers to get involved. Indeed, extension of the database is actively encouraged under the terms of the MIT license (a permissive, free software license which originated at the Massachusetts Institute of Technology). We hope that the user interface and functionality is intuitive and self-explanatory.

\section{AFFILIATIONS}

Centre for Environmental Magnetism \&

Palaeomagnetism, Lancaster University, UK

\section{CONTACT}

Barbara A. Maher: b.maher@lancaster.ac.uk

REFERENCES

Kohfeld KE, Harrison SP (2001) Earth Sci Rev 54: 81-114

Maher BA, Harrison SP (2009) Eos 90: 139

Maher BA et al. (2010) Earth Sci Rev 99: 61-97 